\title{
THE CREATION OF LITHIUM IN GIANT STARS
}

\author{
I.-JULIANA SACKMANN \\ W. K. Kellogg Radiation Laboratory 106-38 \\ California Institute of Technology, Pasadena, CA 91125, USA \\ AND \\ ARNOLD I. BOOTHROYD \\ Canadian Institute for Theoretical Astrophysics \\ University of Toronto, 60 St. George Street, Toronto, Ontario \\ M5S 1A7, Canada
}

A time-dependent "convective diffusion" algorithm for convective transport in the mixing-length framework has been coupled for the first time with a self-consistent full evolutionary computation, in order to investigate theoretically the creation of superrich lithium stars on the asymptotic giant branch. For intermediate mass stars in the mass range from 4 to $7 M_{\odot}$ with both Population I and II compositions, hot bottom burning in the convective envelope was found, with maximum temperatures $T_{\text {ce }}$ at the base of the convective envelope ranging from 20 to 100 million $K$, depending on stellar mass and mass loss rates. For $T_{\text {ce }} \geq 40$ million $\mathrm{K}$, lithium-rich giants were produced (with $\log \varepsilon\left({ }^{7} \mathrm{Li}\right) \gtrsim 1$, i.e., above the normal observed range in giants). For $T_{\mathrm{ce}} \geq 50$ million $\mathrm{K}$, superrich lithium giants were created, with $\log \varepsilon\left({ }^{7} \mathrm{Li}\right) \gtrsim 3$ (i.e., larger than the present $\operatorname{cosmic}{ }^{7} \mathrm{Li}$ abundance). Superrich lithium giants were created for stars in the approximate mass range from 4 to $7 M_{\odot}$ for both Population I and II. Peak ${ }^{7} \mathrm{Li}$ abundances were found to lie in the range $4 \lesssim \log \varepsilon\left({ }^{7} \mathrm{Li}\right) \lesssim 4.6$, relatively independent of mass and chemical composition. We predict a narrow luminosity range for superrich lithium stars, namely $-6 \gtrsim M_{\text {bol }} \gtrsim-7.2$, i.e., $4.3 \lesssim \log L \lesssim 4.8$. Both the predicted peak ${ }^{7} \mathrm{Li}$ abundances and the predicted luminosity range are in beautiful agreement with the observed values for the Galaxy and the Magellanic Clouds. High ${ }^{7} \mathrm{Li}$ abundances persist for $10^{4}$ to $10^{5}$ years. Mass loss in AGB stars can strongly affect the ${ }^{7} \mathrm{Li}$ production; it affects the peak ${ }^{7} \mathrm{Li}$ abundance produced and the mass of lithium-rich material ejected into interstellar space, as well as the timescale and luminosity range over which 
the superrich lithium phenomenon is observable. For a modest mass loss rate (a Reimers' wind with $\eta=1.4$ ), superrich lithium stars are produced from 4 to $7 M_{\odot}$. For a more realistic intermediate mass loss rate $(\eta=5)$, the $4 M_{\odot}$ star was prevented from becoming a superrich lithium star - it never even became lithium rich; for 5 through $7 M_{\odot}$, the peak ${ }^{7} \mathrm{Li}$ abundance is unaffected by the increased mass loss, but the mass of lithium-rich material ejected into space is greatly increased, and thus the total mass of lithium ejected from these stars increases by a factor of 3 over our modest mass loss case. For an extreme mass loss rate $(\eta=14)$, even the $5 M_{\odot}$ star barely reaches superrich lithium abundances $\left(\log \varepsilon\left({ }^{7} \mathrm{Li}\right) \approx 3\right.$ ), ejecting only minor amounts of lithium; on the other hand, the peak ${ }^{7} \mathrm{Li}$ abundance in 6 and $7 M_{\odot}$ stars is unaffected, and the amount of lithium ejected by these stars is again increased, by a factor of 3 over the intermediate mass loss case. We conclude that intermediate mass AGB stars are major sources of cosmic lithium, able to account for $0.5_{+0.5}^{-0.25}$ of the cosmic abundance with our most realistic mass loss rate $(\eta=5)$. With the extreme mass loss case $(\eta=14)$, AGB stars can also provide $0.5_{+0.5}^{-0.25}$ of the cosmic lithium, while the modest mass loss rate $(\eta=1.4)$ can provide $0.2_{+0.2}^{-0.1}$ of the cosmic lithium. 